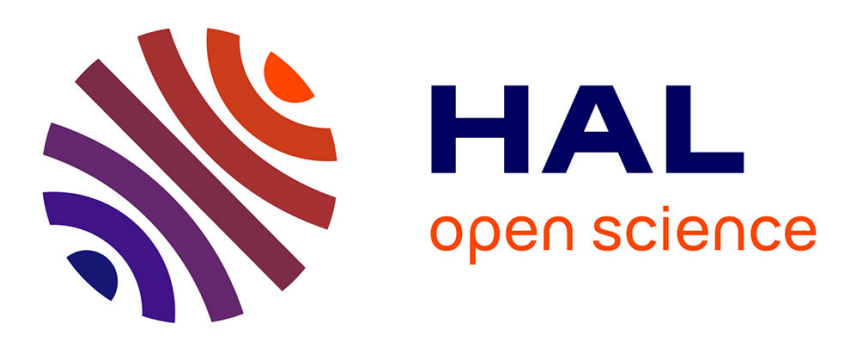

\title{
A geometrical characterization of a class of 0 -flat affine dynamical systems
}

\author{
Soraya Bououden, Driss Boutat, Jean-Pierre Null Barbot, Frédéric Kratz
}

\section{To cite this version:}

Soraya Bououden, Driss Boutat, Jean-Pierre Null Barbot, Frédéric Kratz. A geometrical characterization of a class of 0-flat affine dynamical systems. ACC 2009 - IEEE American Control Conference, IEEE, Jun 2009, Saint Louis, Missouri, United States. 10.1109/ACC.2009.5160122 inria-00408226

\section{HAL Id: inria-00408226 https://hal.inria.fr/inria-00408226}

Submitted on 29 Jul 2009

HAL is a multi-disciplinary open access archive for the deposit and dissemination of scientific research documents, whether they are published or not. The documents may come from teaching and research institutions in France or abroad, or from public or private research centers.
L'archive ouverte pluridisciplinaire HAL, est destinée au dépôt et à la diffusion de documents scientifiques de niveau recherche, publiés ou non, émanant des établissements d'enseignement et de recherche français ou étrangers, des laboratoires publics ou privés. 


\title{
A geometrical characterization of a class of 0 -flat affine dynamical systems
}

\author{
S. Bououden, Driss Boutat, Jean-Pierre Barbot and Frédéric Kratz .
}

\begin{abstract}
This paper gives a description of a class of 0 flat dynamical systems. This class is characterized by the involutivity of a distribution associated naturally to multioutput affine dynamical systems and the Lie bracket of some control vector fields fulfilling some conditions. We will also show that these conditions are a generalization of the well-known result on 0 -flatness of codimension 1 affine systems.
\end{abstract}

\section{INTRODUCTION}

One important problem in control theory is to invert dynamical systems in order to compute the inputs required to perform a given task, for example the trajectory planning problem.

One classical solution to this problem is feedback linearization. Necessary and sufficient conditions for static state feedback linearizability were given in ([20], [22],[24]). For dynamic state feedback linearization, several results can be found in (see [2], [5], [6], [18], [19],[33], [28], [31], [34], [37], [38]).

Another approach to solve the trajectory planning problem, is the concept of differential flatness. This concept was first addressed by Fliess, Lévine, Martin, and Rouchon ([11], [13]), using the differential algebra theory.

A second approach to deal with flatness is exterior differential systems where a control dynamical system is regarded as a Pfaffian system on an appropriate jet space ([1], [7], [8], [25], [28], [39]), and flatness is related to absolute equivalence introduced by E. Cartan [4]. Another geometrical approach by means of Lie-Bäcklund equivalence was addressed in ([12], [14], [15], [23]).

Flat systems are a generalization of Linear dynamical systems in the sense that all linear controllable dynamical systems are flat and static feedback linearizable (in Brunovsky's form). In contrast to the feedback linearization, the flatness does not need to convert nonlinear systems into linear ones to design different kinds of feedback laws ${ }^{1}$ [41], [42], [43]. Therefore, when a system is flat, we can use its structure to design control for motion, trajectory generation and stabilization.

A problem in the flatness theory is to give a general criterion for checking flatness and an algorithm to build the

S. Bououden, D.Boutat and F. Kratz are with ENSI-Bourges, Institut PRISME, 88, Bd. Lahitolle, 18020 Bourges Cedex France

J-P. Barbot is with ECS ENSEA, 6 Avenue du Ponceau, 95014 CergyPontoise, and EPI ALIEN, INRIA

${ }^{1}$ Authors express all their gratitude for anonymous reviewers for many precisions on flatness and references on the topic so-called flat outputs in a constructive way. In addition to the fact that feedback linearizable dynamical systems are flat, some results in this direction exist; [5] controllable codimension 1 affine dynamical systems or codimension 2 non holonome dynamical systems are flat.

In [25], the authors gave a characterization of the so-called $k$-flatness with the Cartan-Kähler approach.

In this paper, we will characterize a 0 -flatness of particular classes of affine nonlinear dynamical systems for which we can build the flat outputs in a constructive way. As we will show with some examples, for this particular class, our method presents a new direction to solve the flatness problem.

This paper is outlined as follows. In the next section, we address notations, definition and the problem statement. In section 3 , we give a class of 0 -flat of nonlinear dynamical systems. This class can be seen as a normal form which is structurally 0 -flat. In section 4 , we give the necessary and sufficient geometrical conditions for affine dynamical systems to belong to the described class in section 3 .

\section{DEFINITIONS, NOTATIONS AND PROBLEM STATEMENT}

Let us consider the following class of nonlinear dynamical systems:

$$
\dot{x}=f(x, u)
$$

where $x \in \mathcal{X} \subseteq \mathbb{R}^{n}, u \in \mathcal{U} \subseteq \mathbb{R}^{m}$ and $f$ is a smooth function on $\mathcal{X} \times \mathcal{U}$.

Definition 1: Dynamical system (1) is flat if there exist $m$ functions $y=\left(y_{1}, \ldots, y_{m}\right)$ called the flat outputs such that:

1) $y\left(x, u, \dot{u}, \ldots, u^{\left(r_{1}\right)}\right)$ is a function of state $x$, input $u$, and the derivatives $u^{(i)}$,

2) $x=\varphi\left(y, \dot{y}, \ldots, y^{\left(r_{2}\right)}\right)$ is a function of the flat outputs and their derivatives,

3) $u=\gamma\left(y, \dot{y}, \ldots, y^{\left(r_{2}+1\right)}\right)$ is a function of the flat outputs and their derivatives.

In this paper, we will deal with multi-input affine dynamical systems in the following form:

$$
\dot{x}=f(x)+\sum_{i=1}^{m} g_{i}(x) u_{i}
$$

Without loss of generality, we will assume within this work that:

Assumption 1: $G=\left[g_{1}, \ldots, g_{m}\right]$ is of rank $m$.

We will characterize a class of dynamical systems for which the flat outputs are only functions of states $x$. Thus, 
in point 1 ) of definition 1 we have $y(x)$. This class of dynamical systems is called 0-flat [25].

Among the flat dynamic systems we can quoted the class of controllable linear dynamical systems. Therefore, another class of 0-flat dynamical systems is given by dynamical systems which are linearizable by means of a diffeomorphism and a static feedback. This class was characterized in ([20], [22],[24]).

Finally, recall that a dynamical affine system with $n$ states and $n-1$ inputs is 0 -flat as soon as it is controllable ([23], [30]).

Hereafter, we give another class of dynamical systems which are 0-flat locally.

\section{A CLASS OF 0-FLAT DYNAMICAL SYSTEM}

Let us in this section give a class of affine controllable dynamical systems in the (2) form which are 0-flat. Let $\nu_{1} \geq \nu_{2} \geq \ldots \geq \nu_{m}$ be $m$ integers such that:

$$
\nu_{1}+\ldots+\nu_{m}=n,
$$

and let $r$ an integer such that:

$$
\begin{aligned}
& \nu_{i} \geq 2 \quad \text { if } i \leq r \\
& \nu_{i}=1 \text { if } i>r
\end{aligned}
$$

Let us set

$$
z=\left\{z_{i, j}, 1 \leq j \leq m \text { and } 1 \leq i \leq \nu_{j}\right\}
$$

and consider the following dynamical system:

For $1 \leq j \leq m$ we set

$$
\begin{aligned}
\dot{z}_{i, j} & =z_{i+1, j}+\sum_{l=k}^{m} \alpha_{i, j}^{l}(z) u_{l} \text { if } 1 \leq i \leq \nu_{j}-1 \\
\dot{z}_{\nu_{j}, j} & =a_{j}(z)+\sum_{l=k}^{m} \alpha_{\nu_{j}, j}^{l}(z) u_{l} \text { if } i=\nu_{j}
\end{aligned}
$$

where $k=\min \left\{l ; \quad \nu_{l} \leq i\right\}$, and

functions $a_{j}$ and $\alpha_{i, j}^{l}$ satisfy to the following conditions:

\section{ASSUMPTION 2:}

1) For $1 \leq k \leq m$, functions $a_{k}$ depend only on the following variables:

- $z_{i, j}$ such that $\nu_{j}>\nu_{k}$ and $1 \leq i \leq \nu_{k}+1$

- $z_{i, j}$ such that $\nu_{j} \leq \nu_{k}$ and $1 \leq i \leq \nu_{j}$

2) Functions $\alpha_{i, j}^{k}$ are as follows:

- if $\nu_{k}>i$, then $\alpha_{i, j}^{k}=0$,

- if $\nu_{k} \leq i$, then $\alpha_{i, j}^{k}$ depend only on the following variables

$$
\begin{aligned}
& -z_{s, l} \text { for } 1 \leq s \leq \nu_{l} \text { if } \nu_{l} \leq i \leq \nu_{j} \\
& -z_{s, l} \text { for } 1 \leq s \leq i+1 \text { if } i<\nu_{l} \leq \nu_{j} .
\end{aligned}
$$

$$
\text { - } \alpha_{\nu_{j}, j}^{j} \neq 0 \text { on } \mathcal{X}
$$

Remark 1: a) For a fixed $i$ and for all $1 \leq j \leq m$ dynamics $\left(\dot{z}_{i, j}\right)_{\nu_{j} \geq i}$ depend on the variables:

1) $u_{k}$ for $\nu_{k} \leq i$

2) $z_{s, l}$ for $1 \leq s \leq \nu_{l}$ if $\nu_{l} \leq i \leq \nu_{j}$,

3) $z_{s, l}$ for $1 \leq s \leq i+1$ if $i<\nu_{l} \leq \nu_{j}$.

Thus, for $1 \leq j \leq m$ dynamics $\dot{z}_{1, j}$ depend only on $\left(u_{k}\right)_{\nu_{k}=1},\left(z_{1, l}\right)_{1 \leq l \leq m}$ and $\left(z_{2, l}\right)_{\nu_{l} \geq 2}$.

Dynamics $\left(\dot{z}_{2, j}\right)_{\nu_{j} \geq 2}$ depend only on: $\left(u_{k}\right)_{\nu_{k}=1},\left(u_{k}\right)_{\nu_{k}=2}$, $\left(z_{1, l}\right)_{1 \leq l \leq m},\left(z_{2, l}\right)_{1 \leq l \leq m}$ and $\left(z_{3, l}\right)_{\nu_{l} \geq 3}$.

And so on.

b) We can use the fact that $\alpha_{\nu_{j}, j}^{j} \neq 0$ on $\mathcal{X}$ to have $a_{\nu_{j}}=0$ and $u_{j}=1$. Indeed, we consider the following static feedback:

$$
u_{j}=\frac{1}{\alpha_{\nu_{j}, j}^{j}}\left(v_{j}-a_{\nu_{j}}\right) .
$$

To give a geometrical interpretation of the above conditions, let us give some notations. We set dynamical system (3)-(4) in the following compact form:

$$
\dot{z}=\bar{f}+\sum_{k=1}^{m} \bar{g}_{k} u_{k},
$$

with

$$
\bar{f}=\left(\begin{array}{c}
\bar{f}_{1} \\
\bar{f}_{2} \\
. \cdot \\
. \cdot \\
\bar{f}_{m}
\end{array}\right)
$$

where for $1 \leq j \leq m$ we have:

$$
f_{j}=\left(\begin{array}{c}
z_{2, j} \\
z_{3, j} \\
\cdot . \\
z_{\nu_{j}, j} \\
a_{j}
\end{array}\right) .
$$

And $1 \leq k \leq m$ we set :

$$
\bar{g}_{k}=\left(\begin{array}{c}
\bar{g}_{1}^{k} \\
\bar{g}_{2}^{k} \\
. \ddot{k} \\
\bar{g}_{m}^{k}
\end{array}\right)
$$

where for $1 \leq j \leq m$

$$
\bar{g}_{j}^{k}=\left(\begin{array}{c}
\alpha_{1, j}^{k} \\
\alpha_{2, j}^{k} \\
\ddot{k} \\
\alpha_{\nu_{j}, j}^{k}
\end{array}\right) .
$$

Thanks to condition (2) in assumption 2 , we have for $1 \leq$ $k \leq m$ :

$$
\begin{gathered}
\bar{g}_{j}^{k}=0 \quad \text { if } \quad \nu_{j}<\nu_{k}, \\
\alpha_{i, j}^{k}=0 \quad \text { if } \quad \nu_{k}<i \leq \nu_{j} .
\end{gathered}
$$


Remark 2:

- Let us consider the following distribution:

$\bar{\Delta}=\operatorname{span}\left\{a d_{\bar{f}}^{k} \bar{g}_{i}\right.$, for all $\nu_{i} \geq 2$ and $\left.0 \leq k \leq \nu_{i}-2\right\}$,

which is involutive. In fact its dual codistribution is given by:

$$
\bar{\Delta}^{T}=\operatorname{span}\left\{d z_{1, j}\right\}_{1 \leq j \leq m} .
$$

This is compatible with the form of dynamic (3)-(4) and the fact that functions $a_{j}$ satisfy point (1) of assumption 2 .

- Conditions 2) are equivalent to the following fact: for $1 \leq k \leq m$ with $\nu_{k} \geq 2$ and for indices $l$ such that: $\nu_{l}<\nu_{k}$ we have for $0 \leq s \leq \nu_{k}-\nu_{l}-1$ :

$$
\begin{gathered}
{\left[\bar{g}_{l}, a d_{f}^{\nu_{k}-\nu_{l}-1-s} \bar{g}_{k}\right] \in} \\
\operatorname{span}\left\{a d_{f}^{j} \bar{g}_{i} \text { for } j=0: \nu_{i}-\nu_{l}-s \text { and } j \geq 0\right\} .
\end{gathered}
$$

Remark 3: Using a linear change of coordinates we can assume that: $a_{k}=O^{2}(z)$ and for $\alpha_{i, j}^{k}$ such that $i \neq \nu_{j}$ we have $\alpha_{i, j}^{k}=O^{1}(z)$.

Now, we have the following preliminary result.

Proposition 1: Under assumptions (1-2), dynamical system (3)-(4) is 0-flat and the flat outputs are $\left(z_{1, j}\right)_{1 \leq j \leq m}$ locally.

Proof: By assumption 2, for a fixed $1 \leq s \leq \max _{j=1}^{m}\left(\nu_{j}\right)$ the following set of dynamics:

$$
\left\{\dot{z}_{s, j}, \quad 1 \leq j \leq m \text { with } s \leq \nu_{j}\right\}
$$

depend only on the $\mathfrak{S}_{s}$ set of the following variables :

1) $u_{k}$ for $\nu_{k} \leq i$

2) $z_{s, l}$ for $1 \leq s \leq \nu_{l}$ if $\nu_{l} \leq i \leq \nu_{j}$,

3) $z_{s, l}$ for $1 \leq s \leq i+1$ if $i<\nu_{l} \leq \nu_{j}$.

We will show that $y_{j}=z_{1, j}$ for $1 \leq j \leq m$ are the flat outputs. For this, we start by writing all variables in the $\mathfrak{S}_{1}$ set by means of $y_{j}=z_{1, j}$ and $\dot{y}_{j}=z_{1, j}$. However, in $\mathfrak{S}_{1}$ we already know the variables $\left(y_{j}=z_{1, j}\right)_{1 \leq j \leq m}$. We then have to determine all the state variables $\left(z_{2, j}\right)_{\nu_{j} \geq 2}$ and outputs $\left(u_{k}\right)_{\nu_{k}=1}$.

- For this, we use the implicite function theorem to compute variables $\left(z_{2, j}\right)_{\nu_{j} \geq 2}$ and the inputs $\left(u_{k}\right)_{\nu_{k}=1}$, from the dynamics $\dot{z}_{1, l}$ for $1 \leq l \leq m$. In fact from the following equations:

$$
\begin{aligned}
& \dot{z}_{1, j} \quad-\quad z_{2, j}-\sum_{l=r+1}^{m} \alpha_{1, l}^{j} u_{l}=0 \text { if } \nu_{j} \geq 2 \\
& \dot{z}_{1, j} \quad-\quad a_{j}-\sum_{l=r+1}^{m} \alpha_{1, l}^{j} u_{l}=0 \text { if } \quad \nu_{j}=1,
\end{aligned}
$$

where $\alpha_{1, l}^{j}$ depend only on $\left(z_{1, l}\right)_{1 \leq l \leq m}$ and $\left(z_{2, l}\right)_{\nu_{l} \geq 2}$. Therefore, we have to compute $m$ variables $\left(z_{2, l}\right)_{\nu_{l} \geq 2}$, $\left(u_{j}\right)_{\nu_{j}=1}$. The differential of the above equation by means of $\frac{\partial}{\partial\left(z_{2, j}, u_{k}\right)}$ is equal to $I+O^{1}(z, u)$ which is locally invertible (here we used point $(b)$ of remark 1 and remark 3).

Therefore, we have :

$$
\begin{aligned}
z_{2, l} & =\varphi_{l}\left(y_{k}, \dot{y}_{k}\right) \text { for } \nu_{l} \leq 2 \\
u_{k} & =\gamma_{k}\left(y_{k}, \dot{y}_{k}\right) \text { for } \nu_{k}=1
\end{aligned}
$$

where $y_{k}=z_{1, k}$ and $1 \leq k \leq m$. Thus, we know all the variables in $\mathfrak{S}_{1}$.

- Second step, we put expressions (6)-(7) in the dynamics $\left(\dot{z}_{2, j}\right)_{\nu_{j} \geq 2}$. Then, we use the same argument to compute $\left(z_{3, l}\right)_{\nu_{l} \geq 3}$ and $u_{k}$ for indices $k$ such that $\nu_{k}=2$ (if $\nu_{k}>2$ we only have to compute $\left.\left(z_{3, l}\right)_{\nu_{l} \geq 3}\right)$. Thus, we know all the variables in $\mathfrak{S}_{2}$

- Then, by induction we assume that we had computed $\mathfrak{S}_{s}$, and from dynamics $\left(\dot{z}_{s+1, j}\right)_{s+1 \leq \nu_{j}}$ we will compute the variables in $\mathfrak{S}_{s+1}$ by using the same argument

Let us give an example of dynamical systems which are in the 0-flat form (3)-(4), to show the procedure of computation of the state variables and the inputs by means of the flat outputs and their derivatives.

Example 1: Consider the following dynamical system :

$$
\left\{\begin{array}{c}
\dot{z}_{1,1}=z_{2,1} \\
\dot{z}_{2,1}=z_{3,1}+\frac{z_{2,1}}{z_{2,2}-1} u_{2} \\
\dot{z}_{3,1}=\left(z_{2,2}-1\right) u_{1}+\frac{z_{3,1}}{z_{2,2}-1} u_{2} \\
\dot{z}_{1,2}=z_{2,2} \\
\dot{z}_{2,2}=u_{2}
\end{array}\right.
$$

We will give the procedure to compute all variable states and inputs from $y_{1}=z_{1,1}$ and $y_{2}=z_{1,2}$. For this, let us consider the following sub-dynamics:

$$
\left\{\begin{array}{l}
\dot{z}_{1,1}-z_{2,1}=0 \\
\dot{z}_{1,2}-z_{2,2}=0
\end{array}\right.
$$

we obtain $z_{2,1}=\dot{y}_{1}$ and $z_{2,2}=\dot{y}_{2}$.

Now, from the following dynamics:

$$
\left\{\begin{array}{c}
\dot{z}_{2,1}=z_{3,1}+\frac{z_{2,1}}{z_{2,2}-1} u_{2} \\
\dot{z}_{2,2}=u_{2}
\end{array}\right.
$$

we obtain: $u_{2}=\ddot{y}_{2}$ and $z_{3,1}=y_{1}^{(2)}-\frac{\dot{y}_{1}}{\dot{y}_{2}-1} \ddot{y}_{2}$.

Finally, from the third equation of the dynamical system we obtain:

$$
\dot{z}_{3,1}-\left(z_{2,2}-1\right) u_{1}-\frac{z_{3,1}}{z_{2,2}-1} u_{2}=0
$$

we obtain

$$
u_{1}=\frac{1}{\dot{y}_{2}-1}\left(y_{1}^{(3)}+\left(\frac{\dot{\hat{y_{1}}}}{\dot{y_{2}-1}} \ddot{y}_{2}\right)-\frac{y_{1}^{(2)}-\frac{\dot{y}_{1}}{\dot{y}_{2}-1} \ddot{y}_{2}}{\dot{y}_{2}-1} y_{2}^{(2)}\right) \text {. }
$$




\section{MAIN RESULT}

In this section, we will give the geometrical necessary and sufficient conditions for the existence of a local diffeomorphism which transforms an affine dynamical system in (2) form into the (3)-(4) form.

For this, we assume that there exist $\nu_{1} \geq \nu_{2} \geq \ldots \geq \nu_{m}$ integers such that :

1) $\sum_{i=1}^{m} \nu_{i}=n$

2) $\Delta_{0}=\left\{a d_{f}^{k} g_{i}\right.$ for $i=1: m$ and $\left.1 \leq k \leq \nu_{k}-1\right\}$ is of rank $n$ on $\mathcal{X}$.

Let us also consider the following distribution:

$$
\Delta=\operatorname{span}\left\{a d_{f}^{k} g_{i}, \text { for all } \nu_{i} \geq 2 \text { and } 0 \leq k \leq \nu_{i}-2\right\}
$$

Theorem 1: There exists a local diffeomorphism which transforms dynamical system (2) into the (3)-(4) form if and only if

1) $\Delta$ is involutive and

2) for $1 \leq k \leq m$ with $\nu_{k} \geq 2$ and for indices $l$ such that: $\nu_{l}<\nu_{k}$ we have for $0 \leq s \leq \nu_{k}-\nu_{l}-1$ :

$$
\left[g_{l}, a d_{f}^{\nu_{k}-\nu_{l}-1-s} g_{k}\right] \in
$$

$\operatorname{span}\left\{a d_{f}^{j} g_{i}\right.$ for $j=0: \nu_{i}-\nu_{l}-s$ and $\left.\nu_{i}-\nu_{l} \geq s\right\}$.

Before giving the proof of the theorem below, let us state the following result.

Corollary 1: If $\nu_{j} \leq 2$ for all $j=1: m$ then there exists a local diffeomorphism which transforms dynamical system (2) into the (3)-(4) form if and only if the distribution

$$
\Delta=\left\{g_{j} \text { for } 1 \leq j \leq m \text { such } \nu_{j}=2\right\}
$$

is involutive. (Thus, we do not need condition (2) of theorem $1)$.

In particular, a codimension 1 dynamical system $m=n-1$ is flat (well-known result [5].)

Remark 4: In the case of a single input $m=1$, we only have condition (1) of theorem 1 and this condition is equivalent to the linearization by means of a diffeomorphism and a static feedback.

Now, we will prove theorem 1.

Proof: Conditions (1)-(2) of theorem 1 are necessary as we showed in remark 2 .

Let us show that these conditions are sufficient. For this, we assume that $\nu_{i} \geq 2$ for $i=1: r$ and $\nu_{i}=1$ of $r+1 \leq i \leq m$. Thus $\operatorname{dim} \Delta=\nu_{1}+\ldots+\nu_{r}-r$ and it is of codimension $m$.

If $\Delta$ is involutive then, there exist $m$ independent functions $h_{1}, \ldots, h_{r}, h_{r+1}, \ldots, h_{m}$ such that:

1) $d h_{i}(\Delta)=0$ for $1 \leq i \leq m$,

2) $d h_{i}\left(a d_{f}^{\nu_{i}-1} g_{i}\right) \neq 0$ on $\mathcal{X}$ for $1 \leq i \leq m$.

Now, let us consider the following coordinates:

$$
z_{i, j}=L_{f}^{i-1} h_{j} \text { for } j=1: m \text { and } 1 \leq i \leq \nu_{i} .
$$

and set $z=\left(z_{j}\right)_{1 \leq j \leq m}$ where for $1 \leq j \leq m$

$$
z_{j}=\left(z_{i, j}\right)_{1 \leq i \leq \nu_{j}}
$$

We consider the diffeomorphism $z=\phi(x)$, and for $1 \leq s \leq m$, we denote by $\overline{g_{s}}=\phi_{*} g_{s}, \overline{g_{s}}=\left(\alpha_{j}^{s}\right)_{1 \leq j \leq m}$ where $\alpha_{j}^{s}=\left(\alpha_{i, j}^{s}\right)_{1 \leq i \leq \nu_{s}}$.

By definition of the new coordinates for $1 \leq j \leq m$ and $1 \leq i \leq \nu_{j}$, we have :

$d z_{i, j} \overline{g_{s}}=0$ for $\nu_{s}-i>0$. Thus, $\alpha_{i, j}^{s}=0$ for $1 \leq j \leq m$ and $1 \leq i \leq \nu_{j}$ such that $\nu_{s}-i>0$.

It is clear that $\phi_{*} f$ is in (3)-(4) form.

Moreover, by the involutivity condition functions $a_{k}$ fulfill points (1) of assumption 2.

Now, the (2) following conditions of theorem:

$$
\begin{gathered}
{\left[g_{l}, a d_{f}^{\nu_{k}-\nu_{l}-1-s} g_{k}\right] \in} \\
\operatorname{span}\left\{a d_{f}^{j} g_{i} \text { for } j=0: \nu_{i}-\nu_{l}-s \text { and } \nu_{i}-\nu_{l} \geq s\right\},
\end{gathered}
$$

implies that $\alpha_{p, q}^{l}$ with $p \leq \nu_{q}$ do not depend on variables $z_{\nu_{l}+s+1, k}$ for $p \leq \nu_{l}+s$. Therefore, point (2) of assumption 2 is fulfilled.

\section{Case 1: Codimension 2 case}

Let us analyse the codimension 2 case, thus $m=n-2$.

By reordering $\left(g_{j}\right)_{1 \leq j \leq m}$ we have two cases :

1) $\nu_{1}=2$ and $\nu_{2}=2$

2) $\nu_{1}=3$.

The first case is similar to corollary 1. Thus, we have to check the involutivity of distribution $\Delta=\operatorname{span}\left\{g_{1}, g_{2}\right\}$.

For the second case, we have to check two conditions :

- distribution $\Delta=\left\{g_{1}, a d_{f} g_{1}\right\}$ is involutive, and

- for all $2 \leq k \leq m$ we must have $\left[g_{k}, g_{1}\right] \in$ $\operatorname{span}\left\{g_{1}, a d_{f} g_{1}\right\}$.

Consider the following academic example [25] modified for a regularity question.

Example 2: Consider the following dynamical system:

$$
\left\{\begin{array}{c}
\dot{x}_{1}=x_{2}+x_{4} x_{3} \\
\dot{x}_{2}=x_{4} \\
\dot{x}_{3}=x_{5} \\
\dot{x}_{4}=u_{1} \\
\dot{x}_{5}=u_{2}
\end{array}\right.
$$

A simple calculation shows that distribution $\Delta_{0}$ is spanned by the following vector fields:

$$
\begin{gathered}
g_{1}=\frac{\partial}{\partial x_{4}}, \\
a d_{f} g_{1}=-\frac{\partial}{\partial x_{2}}-x_{3} \frac{\partial}{\partial x_{1}} \\
a d_{f}^{2} g=\left(1-x_{5}\right) \frac{\partial}{\partial x_{1}} \\
g_{2}=\frac{\partial}{\partial x_{5}} \text { and } a d_{f} g_{2}=-\frac{\partial}{\partial x_{3}} .
\end{gathered}
$$


Thus, $\operatorname{dim} \Delta_{0}=5$ on an open set of 0 such that $x_{5} \neq 1$. Moreover, distribution

$$
\Delta=\operatorname{span}\left\{g_{1}, a d_{f} g_{1}, g_{2}\right\}
$$

is involutive. Thus, condition (1) of theorem 1 is fulfilled. Condition (2) is obviously fulfilled, because $g_{2}$ commutes with $g_{1}$ and $a d_{f} g_{1}$ by means of Lie bracket, thus:

$$
\left[g_{2}, g_{1}\right]=\left[g_{2}, a d_{f} g_{1}\right]=0 .
$$

Now, we will give the diffeomorphism. For this, it is easy to see that codistribution $\Delta^{T}$ is spanned by $d h_{1}$ and $d h_{2}$ where:

$h_{1}=x_{3} x_{2}-x_{1}$ and $h_{2}=x_{3}$.

Therefore, the following diffeomorphism :

$$
\begin{gathered}
z_{1,1}=h_{1} \\
z_{2,1}=L_{f} h_{1}=\left(x_{5}-1\right) x_{2} \\
z_{3,1}=L_{f}^{2} h_{1}=\left(x_{5}-1\right) x_{4} \\
z_{1,2}=h_{2} \\
z_{2,2}=L_{f} h_{2}=x_{5}
\end{gathered}
$$

transforms the dynamical system into the following 0-flat form studied in example 1 :

$$
\left\{\begin{array}{c}
\dot{z}_{1,1}=z_{2,1} \\
\dot{z}_{2,1}=z_{3,1}+\frac{z_{2,1}}{z_{2,2}-1} u_{2} \\
\dot{z}_{3,1}=\left(z_{2,2}-1\right) u_{1}+\frac{z_{3,1}}{z_{2,2}-1} u_{2} \\
\dot{z}_{1,2}=z_{2,2} \\
\dot{z}_{2,2}=u_{2}
\end{array}\right.
$$

Remark 5: If, instead of the first dynamic $\dot{x}_{1}=x_{2}+$ $x_{4} x_{3}$ we take the same dynamic $\dot{x}_{1}=x_{4} x_{3}$ as in [25], then, $\Delta_{0}$ is of rank 5 on an open dense of 0 . In this case, the same flat outputs work well except that $d y_{1}\left(a d_{f}^{3} g_{1}\right) \neq 0$ and $d y_{2}\left(a d_{f}^{2} g_{2}\right) \neq 0$ on an open dense subset.

We think that we can generalize theorem 1 by assuming that distribution $\Delta_{0}$ is of dimension $n$ in a dense subset of $\mathcal{X}$ and $\Delta$ is regular on $\mathcal{X}$.

Let us give another example to highlight the second conditions in theorem.

Example 3: Consider in $\mathbb{R}^{6}$ the following dynamical system:

$$
\left\{\begin{array}{c}
\dot{x}_{1}=x_{2}+\beta u_{2}+\left(\left(1+x_{3}\right) \beta+x_{5}\right) u_{3} \\
\dot{x}_{2}=x_{3}+x_{4} u_{2}+x_{3} u_{3} \\
\dot{x}_{3}=u_{1} \\
\dot{x}_{4}=x_{5} \\
\dot{x}_{5}=u_{2}+x_{3} u_{3} \\
\dot{x}_{6}=x_{3} x_{5} e^{x_{4}}+e^{x_{4}} u_{1}+u_{3}
\end{array}\right.
$$

where $\beta=x_{6}-x_{3} e^{x_{4}}$.
The generators of the distribution $\Delta_{0}$ are:

$$
\begin{aligned}
g_{1} & =\frac{\partial}{\partial x_{3}}+e^{x_{4}} \frac{\partial}{\partial x_{6}} \\
a d_{f} g_{1} & =-\frac{\partial}{\partial x_{2}}, a d_{f}^{2} g_{1}=\frac{\partial}{\partial x_{1}} \\
g_{2} & =\frac{\partial}{\partial x_{5}}+\beta \frac{\partial}{\partial x_{1}}+x_{4} \frac{\partial}{\partial x_{2}} \\
a d_{f} g_{2} & =-\frac{\partial}{\partial x_{4}}-x_{3} e^{x_{4}} \frac{\partial}{\partial x_{6}}+x_{5} \frac{\partial}{\partial x_{2}} \\
g_{3} & =\frac{\partial}{\partial x_{6}}+x_{3} \frac{\partial}{\partial x_{5}}+x_{3} \frac{\partial}{\partial x_{2}}+\left(x_{5}+\left(1+x_{3}\right) \beta\right) \frac{\partial}{\partial x_{1}} .
\end{aligned}
$$

It is easy to see that:

$$
\left[g_{2}, g_{1}\right]=0 \in \operatorname{span}\left\{g_{1}, a d_{f} g_{1}\right\}
$$

$$
\left[g_{3}, g_{1}\right]=-\frac{\partial}{\partial x_{2}}-\frac{\partial}{\partial x_{5}}-\beta \frac{\partial}{\partial x_{1}} \in \operatorname{span}\left\{g_{1}, a d_{f} g_{1}, g_{2}\right\}
$$

Thus, conditions (2) of theorem 1 are fulfilled.

Condition (1) of theorem is also fulfilled. In fact, distribution

$$
\Delta=\operatorname{span}\left\{g_{1}, a d_{f} g_{1}, g_{2}\right\},
$$

is involutive. Moreover,

$$
\Delta^{T}=\operatorname{span}\left\{d h_{1}, d h_{2}, d h_{3}\right\} .
$$

where $h_{1}=x_{1}-x_{5}\left(x_{6}-x_{3} e^{x_{4}}\right), h_{2}=x_{4}$ and $h_{3}=$ $x_{6}-x_{3} e^{x_{4}}$.

Let us set $z_{1,1}=h_{1}, z_{1,2}=h_{2}$ and $z_{1,3}=h_{3}$, we obtain the following diffeomorphism:

$$
\begin{aligned}
& z_{1,1}=x_{1}-x_{5}\left(x_{6}-x_{3} e^{x_{4}}\right) \\
& z_{2,1}=L_{f} h_{1}=x_{2} \text { and } z_{3,1}=L_{f}^{2} h_{1}=x_{3} \\
& z_{1,2}=h_{2}=x_{4} \text { and } z_{2,2}=L_{f} h_{2}=x_{5} \\
& z_{1,3}=h_{3}=x_{6}-x_{3} e^{x_{4}}
\end{aligned}
$$

which transforms the dynamic into the following 0 -flat form (3)-(4):

$$
\left\{\begin{array}{c}
\dot{z}_{1,1}=z_{2,1}+\left(z_{2.2}+z_{1,3}\right) u_{3} \\
\dot{z}_{2,1}=z_{3,1}+z_{1,2} u_{2}+z_{3,1} u_{3} \\
\dot{z}_{3,1}=u_{1} \\
\dot{z}_{1,2}=z_{2.2} \\
\dot{z}_{2,2}=u_{2}+z_{3.1} u_{3} \\
\dot{z}_{1,3}=u_{3}
\end{array}\right.
$$

\section{CONCLUSION}

This paper deals with a characterization of a class of 0 -flat dynamical systems. The conditions fulfilled by this class appear as a natural generalization of conditions of codimension 1 dynamical systems.

In our futur work, we try to characterize a class of $k$-flat dynamical systems by adapting the Charlet, Lévine and Marino method introduced for dynamic feedback linearization in [5]. Acknowledgement

We thank Mr Michel Fliess for all the papers and references that he gave us in the field of flatness and also for his encouragements to work in this field. We also express all our gratitude for anonymous reviewers 


\section{REFERENCES}

[1] R.L. Anderson and N. H. Ibragimov. Lie-Bäcklund Transformations in Applications. SIAM, Philadelphia, 1979.

[2] Aranda-Bricaire E. , C. H. Moog, and J. B. Pomet. A linear algebraic framework for dynamic feedback linearization. IEEE Trans. Automat. Control, 40:127132, 1995.

[3] R. Briant, S. Chern, R. Gardner, H. Goldschmidt, and P. Griffiths. Exterior Differential Systems. Springer Verlag, 1991.

[4] E. Cartan. Sur lequivalence absolue de certains syst'emes dequations differentielles et sur certaines familles de courbes. Bull. Soc. Math. France, 42:1248, 1914. in Oeuvres Compl 'etes, part II, vol 2, pages 11331168, CNRS, Paris, 1984.

[5] B. Charlet, J. Lévine. On dynamic feedback linearization. Systems Control Lett., 13:143151, 1989.

[6] B. Charlet, J. Lévine, and R. Marino. Sufficient conditions for dynamic state feedback linearization. SIAM J. Control Optim., 29:3857, 1991.

[7] E. Delaleau and P. S. Pereira da Silva. Filtrations in feedback synthesis: Part I systems and feedbacks. Forum Math., 10(2):147174, 1998.

[8] J. Descusse and C. H. Moog. Decoupling with dynamic compensation for strong invertible affine nonlinear systems. Internat. J. Control, 42:13871398, 1985.

[9] M. D. Di Benedetto, J. W. Grizzle, and C. H. Moog. Rank invariants of nonlinear systems. SIAM J. Control Optim., 27:658672, 1989.

[10] M. Fliess. Automatique et corps differentiels. Forum Math., 1:227238, 1989.

[11] M. Fliess, J. Lévine, P. Martin, and P. Rouchon. Sur les syst'emes non lineaires differentiellement plats. C. R. Acad. Sci. Paris Ser. I Math., 315:619624, 1992.

[12] M. Fliess, J. Lévine, P. Martin, and P. Rouchon. Linèarisation par bouclage dynamique et transformations de Lie-Bäcklund. C. R. Acad. Sci. Paris Sér. I Math., 317:981986, 1993.

[13] M. Fliess, J. Lévine, P. Martin, and P. Rouchon. Flatness and defect of non-linear systems: introductory theory and examples. Internat. J. Control, 61:13271361, 1995.

[14] M. Fliess, J. Lévine, P. Martin, and P. Rouchon. Deux applications de la geometrie locale des diffiétés. Ann. Inst. H. Poincare Phys. Theor., 66:275292, 1997.

[15] M. Fliess, J. Lévine, P. Martin, and P. Rouchon. Nonlinear control and diffieties, with an application to physics. In J. Krasilshchik M. Henneaux and A. Vinogradov, editors, Secondary Calculus and Cohomological Physics, volume 219 of Contemporary Math., pages 8192, 1998.

[16] M. Fliess, J. Lévine, P. Martin, and P. Rouchon. A Lie-Bäcklund approach to equivalence and flatness of nonlinear systems. IEEE Trans. Automat. Control, 44(5):922937, 1999.

[17] M. Fliess, J. Lévine, P. Martin, and P. Rouchon. Some open question related to flat nonlinear systems. In V.D. Blondel, E. Sontag, M. Vidyasagar, and J.C. Willems, editors, Open Problems in Mathematical Systems and Control Theory, pages 99103, London, 1999. Springer Verlag.

[18] R. B. Gardner and W. F. Shadwick. The GS algorithm for exact linearization to Brunovsky normal form. IEEE Trans. Automat. Control, 37:224230, 1992.

[19] M. Guay, P. J. McLellan, and D.W. Bacon. A condition for dynamic feedback linearization of control-affine nonlinear systems. Internat. J. Control, 68(1):87106, 1997.

[20] L. R. Hunt, R. Su, and G. Meyer. Design for multi-input nonlinear systems. In R. Brocket, R. Millmann, and H. J. Sussmann, editors, Differential Geometric Methods in Nonlinear Control Theory, pages 268298, 1983.

[21] A. Isidori. Nonlinear Control Systems. Springer Verlag, 3nd edition, 1995.

[22] B. Jakubczyk and W. Respondek. On linearization of control systems. Bull. Acad. Pol. Sc., Ser. Sci. Math., 28:517522, 1980.

[23] P. Martin, R. M. Murray, P. Rouchon. Flat Systems. European Control Conference, Plenary Lectures and Mini-Courses, 1997 Brussels.

[24] H. Nijmeijer andW. Respondek. Dynamic input-output decoupling of nonlinear control systems. IEEE Trans. Automat. Control, 33:10651070, 1988.

[25] P. S. Pereira da Silva. Flatness of nonlinear control systems : a Cartan-Kähler approach. In Proc. Mathematical Theory of Networks and Systems MTNS'2000, pages 110, Perpig- nan, Jun. 1923, 2000. CDROM.
[26] P. S. Pereira da Silva. On the nonlinear dynamic disturbance decoupling problem. J. Math. Systems Estim. Control, 6:126, 1996.

[27] J.B. Pomet,(1995). A differential geometric setting for dynamic equivalence and dynamic linearization. Banach Center Publications pp. 319339.

[28] J.B. Pomet. A differential geometric setting for dynamic equivalence and dynamic linearization. In B. Jackubczyk, W. respondek, and T. Rzezuchowski, editors, Geometry in Nonlinear Control and Differential Inclusions, pages 319339, Warsaw, 1995. Banach Center Publications.

[29] J.B. Pomet. On dynamic feedback linearization of four-dimensional affine control systems with two inputs. ESAIM Control Optim. Calc. Var., 2:151230 (electronic), 1997.

[30] F. Rotella and I. Zambettakis Commande des systmes par platitude. Techniques de l'ingènieurs.

[31] P. Rouchon. Necessary condition and genericity of dynamic feedback linearization. J. Math. Systems Estim. Control, 5(3):345358, 1995.

[32] J. Rudolph. Well-formed dynamics under quasi-static state feedback. In B. Jackubczyk, W. Respondek, and T. Rzezuchowski, editors, Geometry in Nonlinear Control and Differential Inclusions, pages 349360, Warsaw, 1995. Banach Center Publications.

[33] W. F. Shadwick. Absolute equivalence and dynamic feedback linearization. Systems Control Lett., 15:35 39, 1990.

[34] William F. Shadwick and Willem M. Sluis. Dynamic feedback for classical geometries. In Differential geometry and mathematical physics (Vancouver, BC, 1993), volume 170 of Contemp. Math. pages 207213. Amer. Math. Soc., Providence, RI, 1994.

[35] S. N. Singh. A modified algorithm for invertibility in nonlinear systems. IEEE Trans. Automat. Control, AC 26:595598, 1981.

[36] W. M. Sluis. A necessary condition for dynamic feedback linearization. Systems Control Lett., 21:277283, 1993.

[37] W. M. Sluis and D. M. Tilbury. A bound on the number of integrators needed to linearize a control system. Systems Control Lett. 29(1):4350, 1996.

[38] D. Tilbury, R. M. Murray, and S. R. Sastry. Trajectory generation for the n-trailer problem using Goursat normal form. IEEE Trans. Automat. Control, 40:802819, 1995.

[39] M. van Nieuwstadt, M. Rathinam, and R. M. Murray. Differential flatness and absolute equivalence of nonlinear control systems. SIAM J. Control Optim., 36(4):12251239 (electronic), 1998.

[40] F. W. Warner. Foundations of differentiable manifolds and Lie Groups. Scott, Foresman and Company, Glenview, Illinois, 1971.

[41] V. Hagenmeyer, E. Delaleau.Robustness analysis of exact feedforward linearization based on differential fatness. Automatica, vol. 39, 19411946.

[42] J. Lévine. On flatness necessary and sufficient conditions. Proc. NOLCOS 2004, Stuttgart (Germany), Sep. 2004.

[43] K. Schlacher, M. Schberl.Construction of flat outputs by reduction and elimination. Proc. NOLCOS 2007, Pretoria (South Africa), Aug. 2004. 